\title{
Terahertz-Range Gyrodevices of Planar Geometry
}

\author{
V.Yu. Zaslavsky, N.S. Ginzburg, I.V. Zotova, M.Yu. Glyavin, \\ A.S. Sergeev, R.M. Rozental, A.M. Malkin, I.V. Zheleznov \\ Institute of Applied Physics of the Russian Academy of Sciences, Niznhny Novgorod, Russia, zas-vladislav@ipfran.ru
}

For drastic increase in the output power of shortwavelength gyrotrons, we suggest to use the planar scheme with a sheet electron beam and transverse (with respect to the electrons translation velocity) electromagnetic energy extraction. The main advantage of this scheme comparing to the conventional cylindrical geometry is the possibility of effective mode selection over the open transverse coordinate in combination with radiation out-coupling, which leads to substantial reduction of Ohmic losses [1,2]. The formation of ribbon helical electron beam (HEB) in planar magnetron-injection gun (MIG) was demonstrated in [3]. In this paper we consider gyrotrons and gyroklystrons of planar geometry driven by sheet electron beams. Possibility of power increasing and selectivity improvement in the terahertz band is shown.

\section{High-Order Cyclotron Harmonic Excitation in a THz-Range Planar Gyrotron}

An additional advantage of the planar scheme is the peculiarity associated with excitation of odd $(s=1,3 \ldots)$ and even $(s=2,4 \ldots)$ cyclotron harmonics. Under assumption that the sheet electron beam is injected in the middle of the cavity between the plates, the interaction at odd cyclotron harmonics occurs only for resonator modes with odd transverse indexes $n=1,3 \ldots$, while interaction at even harmonics occurs only for modes with even transverse indexes $n=2,4 \ldots$. Moreover, for example, for operation at the second harmonic it is beneficial to use an even resonator mode with indexes $n$ equal to doubled even number. In this case interaction at second harmonic will be not accompanied by simultaneous excitation of a lower order mode at the first cyclotron harmonic due to the coupling factor for the $1^{\text {st }}$ harmonic with an even mode is equal to zero. From the other hand, for excitation at the odd cyclotron harmonic the number of the resonator mode should not be dividable by $s$. For example, for $s=3$, the resonator mode number may be $n=5,7,11 \ldots$. In this case the parasitic mode at the $2^{\text {nd }}$ cyclotron harmonic is not excited. At the same time, excitation at the $1^{\text {st }}$ cyclotron harmonic can be suppressed due to sensitivity to the spread of electrons velocity and due to additional diffraction losses caused by some penetration of low-frequency running wave through collector narrowing.

Further results of simulations of $1.2 \mathrm{THz}$ planar gyrotron operating at the $3^{\mathrm{d}}$ cyclotron harmonic are presented. Simulations were carried out in the frame of averaged multi-modes approaches:

$$
\begin{aligned}
& s_{n} \frac{\partial a_{n}}{\partial \tau}+i \frac{\partial^{2} a_{n}}{\partial X^{2}}+i \frac{\partial^{2} a_{n}}{\partial Z^{2}}+\left(i \Delta_{n}+\sigma_{n}\right) a_{n}=\frac{I_{n}}{2 \pi} \int_{0}^{2 \pi} p^{s} d \vartheta_{0}, \\
& \frac{\partial p}{\partial \tau}+\frac{g^{2}}{4} \frac{\partial p}{\partial Z}+i p\left(|p|^{2}-1\right)=-\sum_{n} a_{n}\left(p^{*}\right)^{S-1} .
\end{aligned}
$$

Here $a_{n}$ is the amplitude of the $T E_{n}$ mode excited at the $s_{n}$ harmonic of cyclotron gyrofrequency, $p$ is the transverse electron momentum, $\Delta_{n}$ is the cyclotron resonance detuning, $I_{n}$ is the parameter proportional to electron current and the coupling factor $G_{n}(y)$, $\sigma_{n}$ is the parameter of Ohmic losses proportional to the skin depth. Due to the diffraction extraction of radiation is realized in transverse $x$ direction (Fig.1a), at the edges of interaction space the nonreflecting boundary conditions may be applied. In the direction of translational motion of electrons the waveguide is considered to be closed by cut-off necks. Thus we can apply zero boundary condition along $z$ coordinate.

(a)

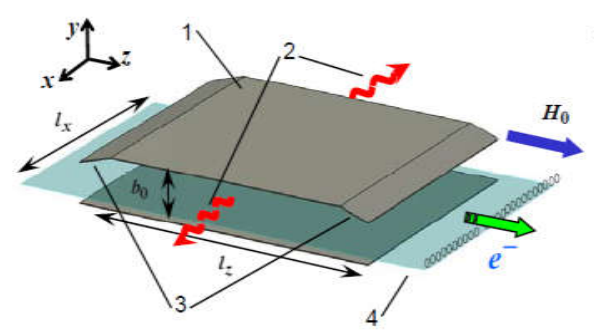

(b)

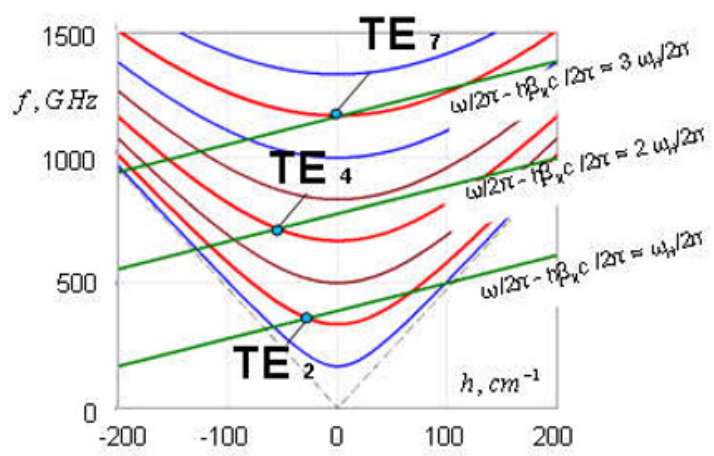

Fig. 1. (a) Scheme of a planar gyrotron with transverse energy extraction: (1) - planar waveguide, (2) - energy output channels, (3) - cutoff narrowing, (4) - sheet helical electron beam. (b) Dispersion diagram of competing modes in the case of exciting the $3^{\mathrm{d}}$ cyclotron harmonic.

Simulations were carried out for following parameters: energy of electrons of $30 \mathrm{keV}$, electron current of $6 \mathrm{~A}$, pitch-factor of 1 , the distance between plates of $0.9 \mathrm{~mm}$, resonator length and width of 
$20 \mathrm{~mm}$ both. Three modes exciting at the $1^{\text {st }}, 2^{\text {nd }}$ and $3^{\mathrm{d}}$ cyclotron harmonics were included in consideration. Results of simulations (see Fig.2) confirm the possibility of selective excitation of the operating $T E_{7}$ mode at the frequency of $1.2 \mathrm{THz}$.

(a)

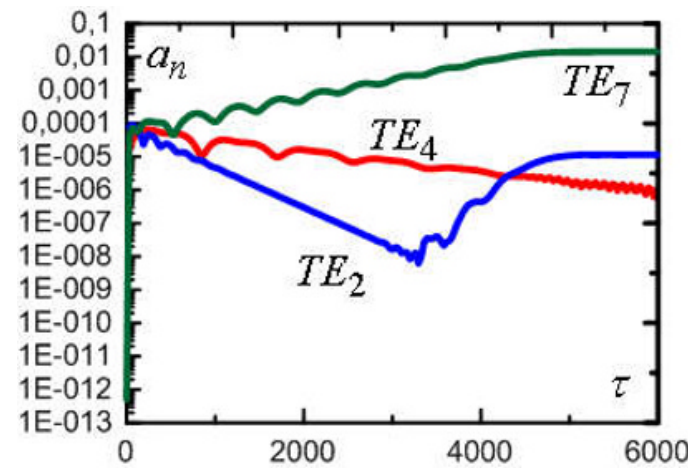

(b)

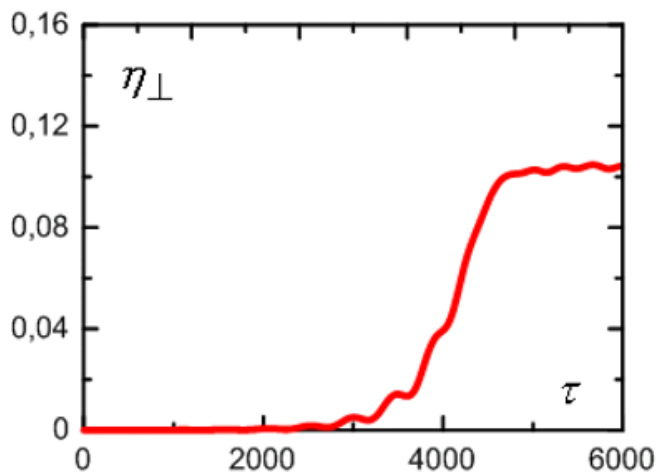

Fig. 2. Temporal dependencies of amplitudes of competing modes (a) and the transverse electron efficiency (b).

\section{Planar Gyro-Klystrons}

Using of planar configuration in gyro-klystrons is of interest as a method of increasing the power of output radiation. The considered scheme is presented in Fig. 3a. Analysis was carried out based on 3D PIC (Particle-in-cell) simulations using the code KARAT. Parameters of electron beam were following: energy of $20 \mathrm{kV}$, total current of $2 \mathrm{~A}$, pitch-factor of 1.3 . For distance between plates of $2.2 \mathrm{~mm}$ and excitation of the $T E_{3}$ mode the operating frequency was of $200 \mathrm{GHz}$. According to simulations for input power of $10 \mathrm{~W}$ output power achieves $4.5 \mathrm{~kW}$. It corresponds to the amplification depth of $26 \mathrm{~dB}$ and efficiency of about $10 \%$. (a)

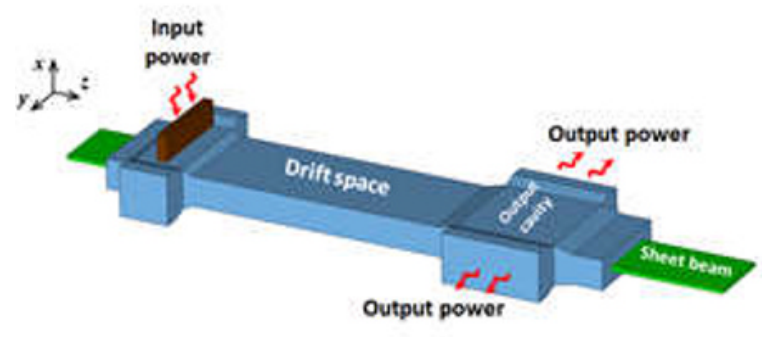

(b)

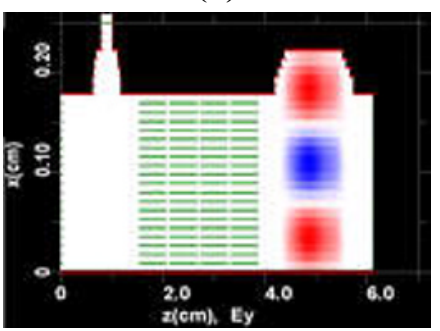

(c)

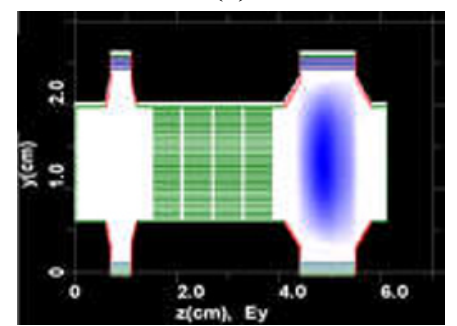

Fig. 3. (a) Scheme of gyroklystron of planar geometry. (b), (c) Field distributions in the input and output resonator over $\mathrm{x}$ and $\mathrm{y}$ coordinates.

The work was supported by the Russian Science Foundation under grant No. 18-19-00704.

\section{References}

1. Ginzburg N.S., Zotova I.V., Sergeev A.S., Zaslavsky V.Yu., Zheleznov I.V., High-power terahertz-range planar gyrotrons with transverse energy extraction// Phys. Rev. Lett. 2012. V. 108. Art.no. 105101.

2. Zaslavsky V.Yu., Ginzburg N.S., Glyavin M.Yu., et al. Three-dimensional particle-in-cell modeling of terahertz gyrotrons with cylindrical and planar configurations of the interaction space. // Physics of Plasmas. 2013. V. 20. P. 043103.

3. Manuilov V.N., Zaslavsky V.Yu., Ginzburg N.S., et al. Electron-optical systems for planar gyrotrons. // Physics of Plasmas. 2014. V. 21. P. 023106. 